PREPRINT November 13, 2018

\title{
The phase plane of moving discrete breathers
}

\author{
Paul A. Houle \\ Laboratory of Atomic and Solid State Physics, Cornell University, Ithaca, NY, 14853-2501
}

\begin{abstract}
We study anharmonic localization in a periodic five atom chain with quadratic-quartic spring potential. We use discrete symmetries to eliminate the degeneracies of the harmonic chain and easily find periodic orbits. We apply linear stability analysis to measure the frequency of phonon-like disturbances in the presence of breathers and to analyze the instabilities of breathers. We visualize the phase plane of breather motion directly and develop a technique for exciting pinned and moving breathers. We observe long-lived breathers that move chaotically and a global transition to chaos that prevents forming moving breathers at high energies.
\end{abstract}

PACS numbers: 03.20.+i,63.20.Ry,63.20.Ls

Typeset using REVTEX 
A body of theoretical work has appeared in the past decade on intrinsic localized modes in perfect anharmonic crystals. [1] Although the existence of breather periodic orbits with localized energy is established [2], less is known about the rest of the phase space. In this paper, we use a periodic chain of five atoms coupled by a quadratic-quartic springs as a model for larger chains and as a testbed for techniques which can be applied to other systems. We use discrete reflection symmetries to find submanifolds on which finding breather solutions is simplified and on which breather solutions can be continued to the phonon limit. We introduce coordinates for visualizing breather motion, exhibiting the movability separatrix introduced in [3], and detecting chaos in the separatrix region which leads to long-lived breathers that move erratically. We also apply linear stability analysis to investigate phononlike excitations in the presence of a breather and to map the stable and unstable manifolds of an unstable breather, developing a technique to systematically launch moving and pinned breathers complementary to existing methods. [5].

Introducing our system, the Hamiltonian is

$$
\begin{gathered}
H=\sum_{i=1}^{N} M \frac{p_{i}^{2}}{2}+\sum_{i=1}^{N} U\left(q_{i}-q_{i-1}\right), N=5 \\
U(x)=\frac{K_{2}}{2} x^{2}+\frac{K_{4}}{4} x^{4} .
\end{gathered}
$$

under condition that $K_{4}>0$. By scaling $q_{i}$ and $p_{i}$ we set $M=K_{2}=K_{4}=1$ without loss of generality. The system has four nontrivial degrees of freedom since center of mass momentum is conserved. To get numerical results we integrate the equations of motion for (1]) by fifth-order Runge-Kutta with a fixed step size of $10^{-3}$. [6] Because a high amplitude breather is localized on a few atoms, it is reasonable that our small model captures much of the physics of larger chains. To check this we transplanted both even and odd breather solutions of the $N=5$ chain at energy $E=3$ onto a $N=20$ chain with all other atoms initially at rest with zero displacement; we found the breather remained localized for over one thousand breather oscillations without obvious loss of amplitude.

We take advantage of discrete symmetries to explore a subspace of the complete phase 
space of the chain; because breather periodic orbits lie on these subspaces, we can use symmetry to find breathers and to follow breather solutions all the way to the $E=0$ phonon limit. The Hamiltonian (1) respects two kinds of reflection symmetry which we refer to as even and odd symmetry after the even-parity and odd-parity breathers in prior literature. 114. The displacements and momenta of an even breather have even parity with respect to reflections around a bond. (the pattern of displacements is roughly $\mathbf{q}=\left(-\frac{1}{6}, 1,-1, \frac{1}{6}, 0\right)$ for a even breather on the bond between atoms 2 and 3). We refer to this property as even symmetry and the submanifold of the phase space with even symmetry as the even manifold. The odd-parity periodic orbit has odd symmetry, odd parity with respect to reflections around an atom. (displacements are roughly $\mathbf{q}=\left(0,-\frac{1}{2}, 1,-\frac{1}{2}, 0\right)$ for an odd breather centered on atom 3) The submanifold of the chain with odd symmetry is the odd manifold. Even and odd symmetry are respected by the dynamics; if the system starts in the even or odd submanifold it remains there for all time. Even and odd manifolds, of course, exist for each site but as the chain is translationally invariant the dynamics are identical at all sites. Even and odd symmetry can be used to simplify any sized chain — the use of symmetry is particularly advantageous for the $N=5$ chain since the even and odd submanifolds are reduced to two degree of freedom systems which are simple to understand and visualize.

Unlike chains with an even number of atoms [7] [8] there is no energy threshold for breathers in a chain with an odd number of atoms. As the energy $E \rightarrow 0$ we expect the anharmonic chain to be approximated by a harmonic chain for short times. For $\mathrm{N}=5$, the harmonic chain has two degenerate pairs of normal modes with wavenumbers $k_{n}=\frac{n \pi}{5}$ and frequencies $\omega_{ \pm 1}=2 \sin \frac{\pi}{5}$ and $\omega_{ \pm 2}=2 \sin \frac{2 \pi}{5}$. The modes with the highest $k$ are band-edge modes. In general, chains with an even number of atoms have a single band edge mode with wavenumber $\pi$ and chains with an odd number of atoms have two degenerate band edge modes. Since any linear combination of two phonons with the same frequency is a periodic orbit of a harmonic chain, each degenerate pair of phonons is associated with a two parameter family of periodic orbits. This degenerate situation is structurally unstable and is 
shattered when the slightest amount of $K_{4}$ is turned on. Since only one linear combination of phonons intersects each submanifold, we can remove the degeneracy by restricting ourselves to an even or odd manifold on which a single parameter family of periodic orbits survives in the anharmonic system. Specifically, a band-edge phonon with even or odd symmetry deforms continuously into an even or odd breather as energy increases. No bifurcation occurs, thus there is no energy threshold for the formation of a breather in an $N$ odd chain. The situation is different from that in $N$ even chains for which there exists a single band-edge mode possessing both even and odd symmetry which undergoes a symmetrybreaking tangent bifurcation at an energy proportional to $N^{-1}$ into two different periodic orbits corresponding to both even and odd breathers. [7] [8]

Poincáre sections are effective for visualizing dynamics on the submanifolds and provide a method of finding breather solutions. Although restriction to submanifolds should simplify the search for breathers for a system of any size, it is very advantageous for $N=5$ where the problem is reduced to a one dimensional root search. Periodic orbits manifest as fixed points of the Poincáre map on the surface of section $q_{3}=0, \dot{q}_{3}>0$. We plot surfaces of section by integrating the equation of motion until the trajectory crosses the surface of section at this point we use Newton-Raphson to solve for the duration of a Runge-Kutta step that lands on the surface of section. Fixed points corresponding to breather periodic orbits lie on the $q_{1}=0$ line and can be found by a simple 1-d root search by the Brent algorithm. [9] Both the even and odd manifolds can be visualized by plotting $\left(q_{1}, p_{1}\right)$; since the submanifolds are two-dimensional, the result is a complete description of the dynamics on a submanifold. Fig. 11 is an example. Chaos is prevalent in odd manifold sections above $E=1$; we have not observed obvious chaos in the even manifold in the range $0<E<300$ that we've studied.

We use surfaces of section with a different method of projection to directly visualize the movability separatrix introduced in [3] in a manner that should be useful for characterizing moving and pinned modes in other breather systems. The phase plane of breather motion at $E=10$ is seen in Fig. 2. The location of the breather is given by the angle $\theta=\operatorname{Arg} h$ where 


$$
h=\sum_{i=1}^{N} \frac{p_{i}^{2}}{2} e^{i \frac{2 \pi}{N} n}+\sum_{i}^{N} U\left(q_{i}-q_{i-1}\right) e^{i \frac{2 \pi}{N}\left(n+\frac{1}{2}\right)},
$$

with $\mathrm{N}=5$. (3) is a complex weighted average of the kinetic and potential energy on each atom and bond that takes in account the circular nature of the chain, a strong influence on small chains. [10] We construct a variable conjugate to $\theta$ by treating $\delta \theta=\theta_{n}-\theta_{n-1}$. as the velocity of the breather. The $q_{3}=0$ trigger works well when a breather is localized in the range $1<\theta<5$. [11] Unlike Fig. 1, Fig. 2 is a projection from a high-dimensional space to the plane and is not a complete picture of the dynamics. The curves in Fig. 2 is consistent with the hypothesis [3] [4] that two sets of action-angle variables exist approximately for breather states; one set connected with the breather's spatial position and velocity and a set of "internal" degree of freedoms associated with breathing. As occurs for the pendulum, the position-velocity conserved surfaces change topology at the separatrix dividing pinned and moving states. Even the slightest degree of nonintegrability breaks conserved surfaces in the separatrix creating a layer of chaos. [12] We refer to the chaos observed in this separatrix as hopping chaos because trajectories within the separatrix region move erratically in space while remaining localized for thousands of oscillations or more as observed in Fig. 3. Hopping breathers have been observed to decay, so it is clear that the hopping chaos region of phase space is connected to delocalized chaotic regions; however, the region of hopping chaos appears to be sufficiently hemmed in by KAM tori that hopping chaos is a distinct intermediate-term behavior. The region of hopping chaos enlarges as energy increases; near $E=20$ a global transition to chaos in the phase plane of Fig 2 appears to occur and it becomes impossible to create moving modes; only the islands of near integrability corresponding to pinning on a bond remain. Hopping chaos is probably less robust in longer chains since longer chains have more degrees of freedom for resonances to occur with and for energy to be radiated into.

Numerical linear stability analysis gives a local picture of the phase space around a periodic orbit complementary to the more global views of previous sections. With stability analysis we examine phonon-like excitations in the presence of breathers and analyze the 
instabilities of breathers. Let us write the state of the system as a phase space vector $\mathbf{x}=(\mathbf{q}, \mathbf{p})$; let $\mathbf{x}_{\mathbf{0}}$ be a point on a periodic orbit with period $T$. We make an infinitesimal change $\delta \mathbf{x}$ in the initial conditions, launching the system at time $t=0$ in state $\mathbf{x}=\mathbf{x}_{0}+\delta \mathbf{x}$. When we observe the system at time $t=T$ the system is in state $\mathbf{x}_{T}=\mathbf{x}_{0}+\delta \mathbf{x}_{T}$. To linear order in $\delta \mathbf{x}$

$$
\delta \mathbf{x}_{T}=S \delta \mathbf{x}_{\mathbf{0}}+O\left(\delta \mathbf{x}_{0}^{2}\right)
$$

where $S$ is the stability matrix.

With an accurately known periodic orbit we can determine $S$ numerically by making copies of the system and perturbing them successively in each position and momentum coordinate and then evolving each system for time $T$. [13] To interpret the stability matrix, we first find eigenvalues and eigenvectors with EISPACK. 99 For a Hamiltonian system $S$ is a symplectic matrix with certain constraints on the eigenvalues and eigenvectors. [12 In our application, eigenvalues values come in three kinds of pairs; elliptic pairs $\lambda=e^{ \pm i \phi}$ indicating phonon-like excitations in the presence of a breather, hyperbolic pairs $\lambda_{1}=\lambda_{2}^{-1}$ with $\lambda_{1}$ real indicating instabilities and parabolic pairs $\lambda_{1}=\lambda_{2}=1$ arising from conserved quantities. In our application two parabolic pairs arise due to conserved quantities; one pair due to conservation of momentum and another due to conservation of energy - these uninteresting pairs are removed by automated inspection of eigenvectors.

Stability analysis confirms that the even breather known to be stable in long chains [14] is linearly stable in the five atom chain; no hyperbolic eigenvalues appear in the energy range from $E=0-200$. If a stable breather is infinitesimally perturbed, one excites phononlike disturbances that we call quasi-phonons; by investigating quasi-phonons one can study the interaction between a breather and phonons, crucial for understanding quantum and thermal fluctuations around breathers. [15] We determine the frequencies of quasi-phonons from eigenvalues of the stability matrix; Fig 1 plots quasi-phonon frequencies as a function of breather energy. To test the accuracy of our technique, we excited quasi-phonons by making a small change in the $E=3$ and $E=50$ breather solutions; quasi-phonon frequencies 
appeared as peaks in the power spectrum determined by running the system until $t=3000$ and taking the FFT of one atom's position as a function of time. The two methods agree to within one part in $10^{-4}$, the bin size of the power spectrum. Quasi-phonon frequencies vary smoothly with breather energy and converge on the true phonon frequencies as the energy of the breather goes to zero. Therefore we label quasi-phonons by the symmetry they uphold and the wavenumber $k_{n}$ of the phonon they become in the $E \rightarrow 0$ limit, a scheme that should remain applicable for quasi-phonons in larger chains and higher dimensions. The $n=2$ quasi-phonon is tangent to the phase plane of breather motion; a small excitation of the $n=2$ quasi-phonon causes the breather to rock around one bond while a large excitation causes the breather to break free and move as has been observed in a $\phi^{4}$ lattice. [5]

Perturbation of a stable breather has been used to create moving breathers in a $\phi^{4}$ lattice [5]; we have found an alternate method of creating pinned and gliding breathers by perturbing an unstable breather. As is known for large chains, [14 the odd breather is linearly unstable for all energies; the linear instability does not cause the breather to decay but instead causes the breather to move. The eigenvectors of $S$ with eigenvalues less than and greater than one point respectively into the stable and unstable manifolds and define a plane tangent to the phase plane of Fig. 2. As the energy of the odd breather goes to zero, the unstable mode eigenvectors converge on the even band-edge phonon. The tangent plane is divided into four quadrants by the stable and unstable manifolds; distinct regular behaviors are observed for perturbations directed into each quadrant as illustrated by the drawn-in separatrix in Figure 2 - the unstable manifolds of an odd breather at one site feed into the stable manifolds of odd breathers at neighboring sites. By choosing a quadrant we can launch a breather that travels either to the left or to right or that remains pinned while rocking slightly to the left or right of the odd breather location. To get reliable results it is necessary to add a sufficiently large perturbation so as to clear the region of hopping chaos in the separatrix region.

In summary, we have found the $N=5$ chain exhibits much of the phenomenology of larger chains and can be used as both a model of localization and a testing ground for techniques. 
We have applied numerical stability analysis to to measure quasi-phonon frequencies in the presence of a breather and to design perturbations that create either moving or pinned modes starting from either a stable or an unstable breather. Chaos exists in the movability separatrix and becomes increasingly prevalent as the energy increases; a global transition to chaos near $E=20$ makes it impossible to launch moving breathers. With appropriate coordinates we have been able to directly visualize the phase plane of breather motion.

I would like to thank C. Henley for suggesting equation (3) and other useful discussions as well as Rongji Lai, S. A. Kiselev and A. J. Sievers. This work was supported by by NSF Grant DMR-9612304. 


\section{FIGURES}

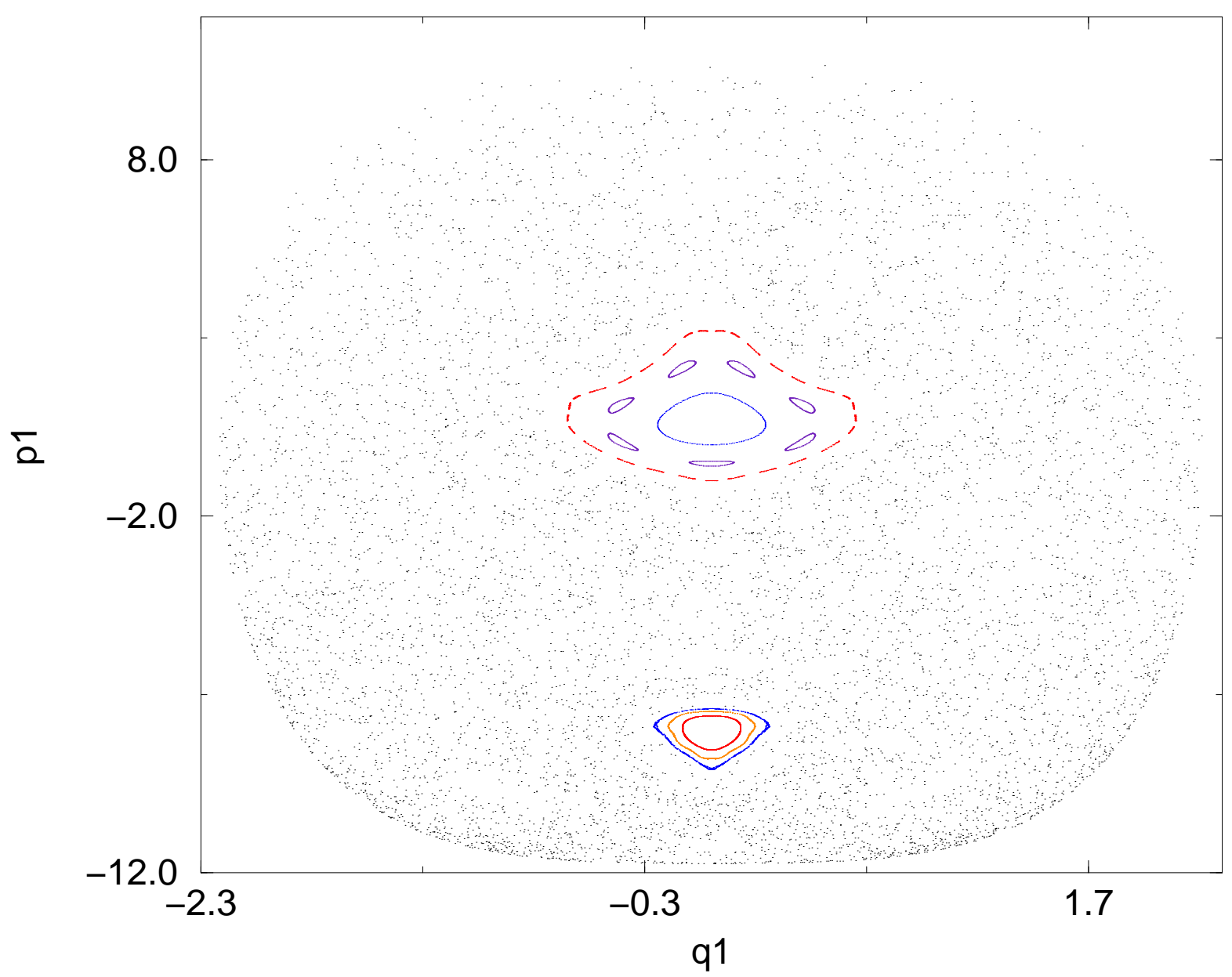

FIG. 1. A Poincáre section on the odd submanifold at $E=230$; the phase space is dominated by chaotic trajectories, although two large islands of regularity are visible. The upper island consists of an unstable breather and phonon-like disturbances around it while the lower island is due to a high-amplitude standing wave associated with the $n= \pm 1$ phonons; resonant islands in the upper half of the plot involve phase locking between the breather and quasi-phonons 


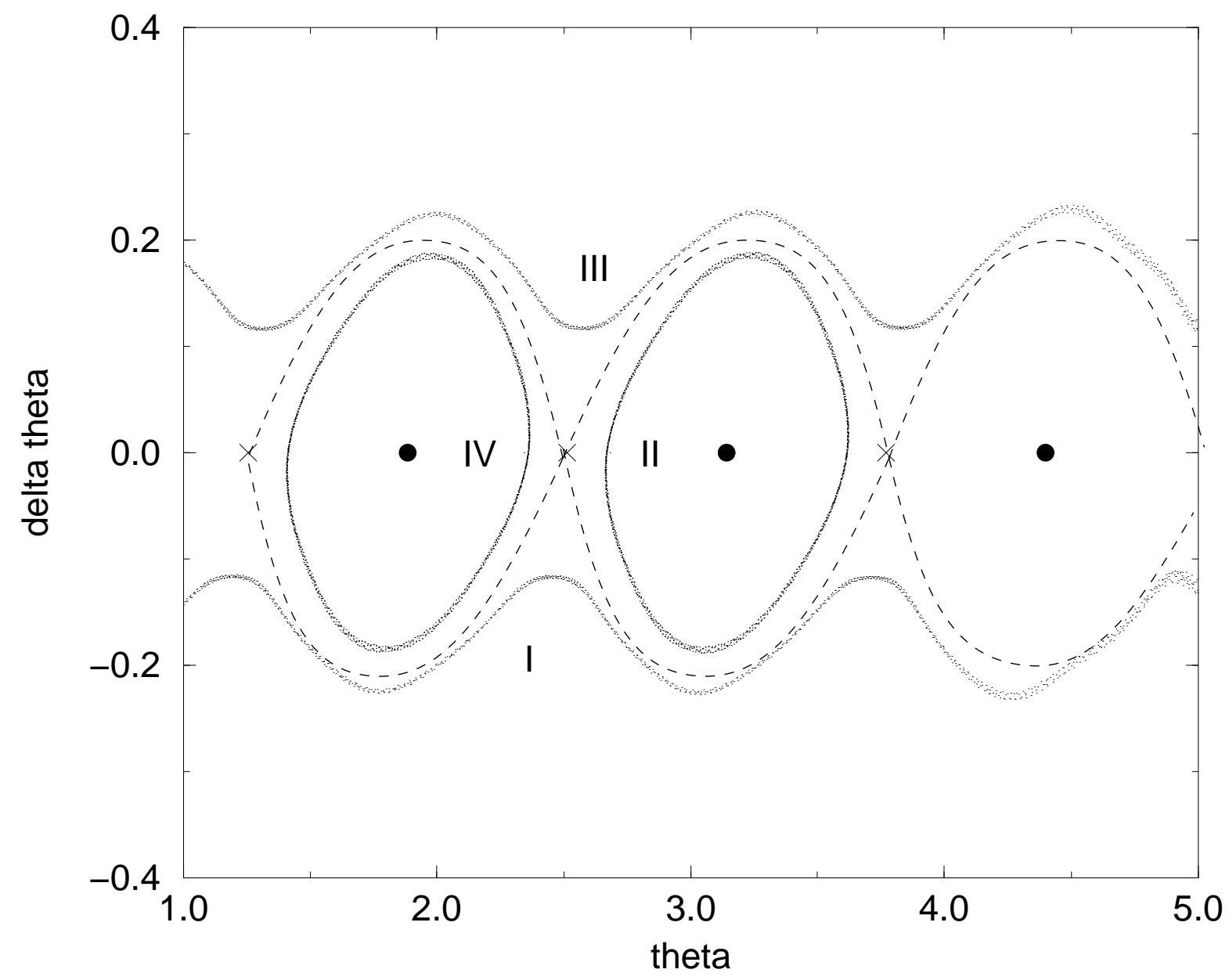

FIG. 2. A Poincáre section using the $q_{3}=0$ trigger and the variable $\theta$ illustrating the pendulum-like phase plane of moving breathers. The dotted line is drawn in and illustrates the separatrix; circles mark stable (even) breather solutions and crosses mark unstable (odd) breather solutions. Roman numerals indicate four regions in phase space divided by stable and unstable manifolds in which distinct regular behaviors are observed. In regions I and III the breather moves to the left and right respectively while it is pinned at either side of site three in regions II and IV. 


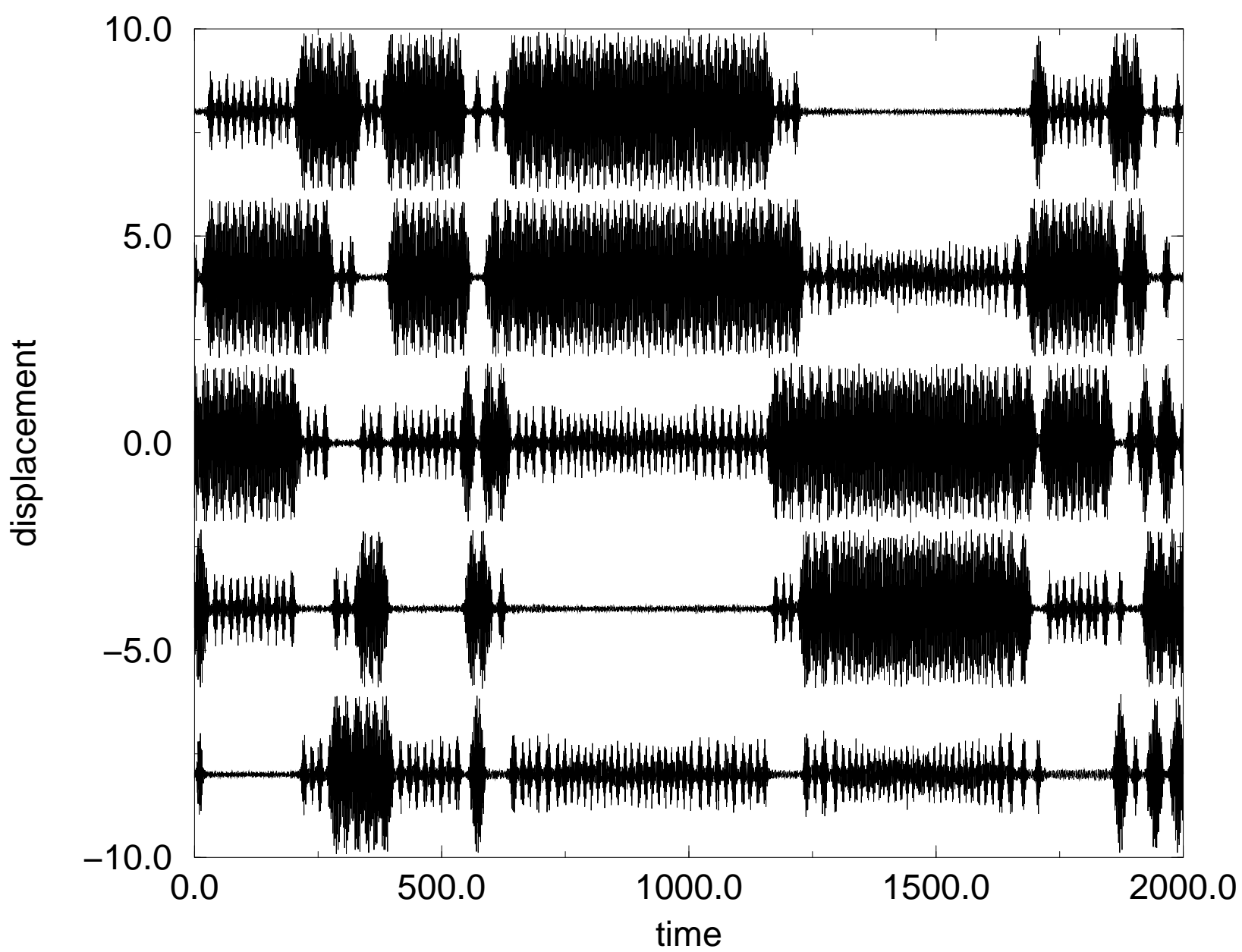

FIG. 3. Hopping chaos observed at $E=50$ for a small perturbation in region III. Although energy is localized throughout the duration of the simulation, the breather moves erratically. The atoms are visually separated by adding constants to the displacements; the chains is periodic so the bottom atom is adjacent to the top atom. 


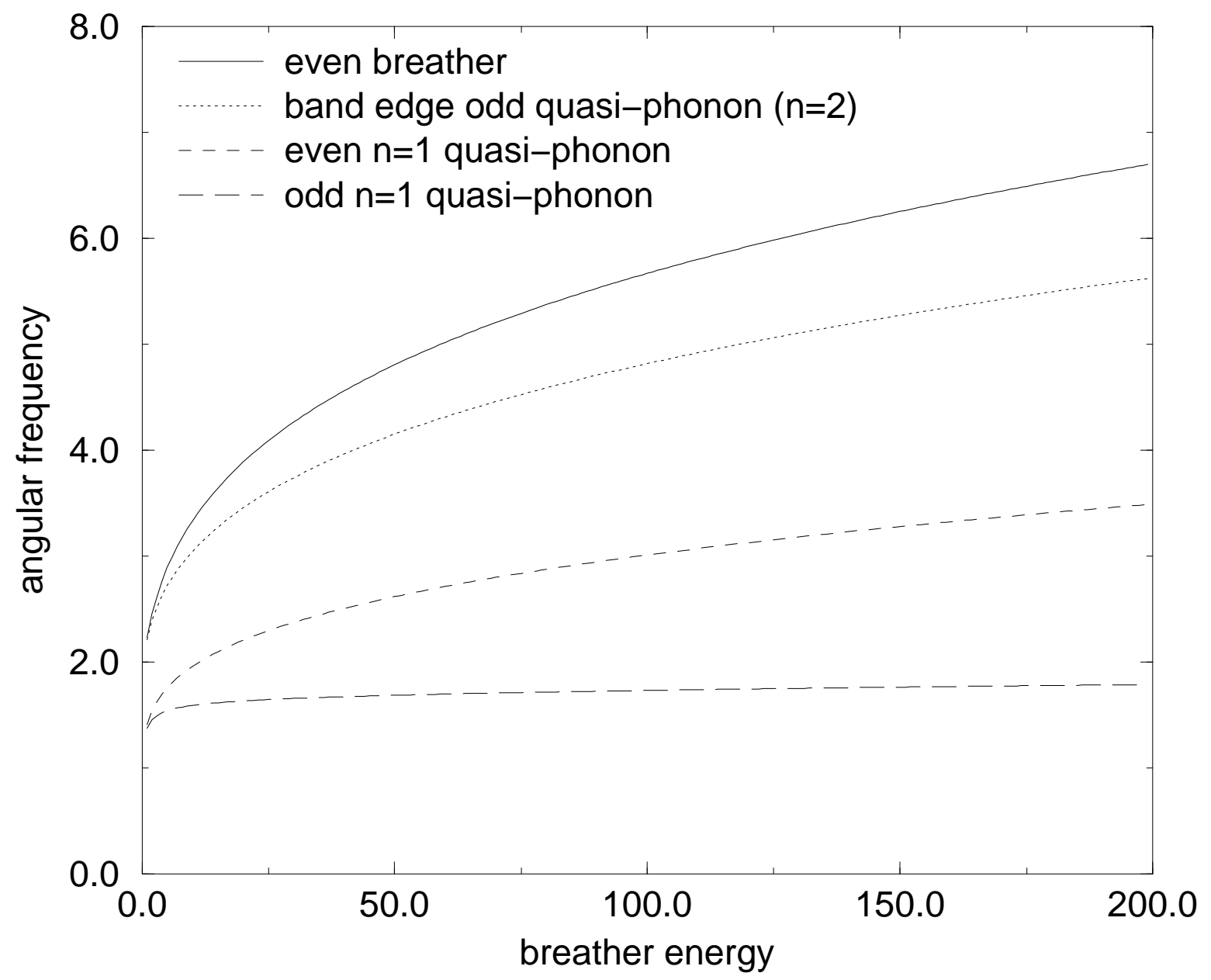

FIG. 4. Breather and quasi-phonon frequencies from the stability matrix for even breathers on a 5 -atom quadratic-quartic chain. The wavenumber of a quasi-phonon is $k= \pm \frac{2 \pi}{5} n$ 


\section{REFERENCES}

[1] S. Flach, C.R. Willis Physics Reports in press (1997)

[2] R.S. MacKay, S. Aubry Nonlinearity 71623 (1994)

[3] S. Flach, C.R. Willis Phys. Rev. Lett. 721777 (1994)

[4] S. Flach, C.R. Willis, E. Olbrich Phys. Rev. E 49836 (1994)

[5] Ding Chen, S. Aubry, G. P. Tsironis Phys. Rev. Lett. 774776 (1996)

[6] W.H. Press, S.A. Teukolsky, W.T. Vetterling, B.P.Flannery Numerical Recipes in C, 2nd edition (Cambridge University Press, New York 1992)

[7] S. Flach Physica D 91223 (1996)

[8] K.W. Sandusky, J.B. Page Phys. Rev. B 50866 (1994)

$[9]$ http://www.netlib.org/

[10] A similar variable applicable only for large chains is used in [3]

[11] Triggering fails near $\theta=0$; perfect triggering is difficult since it is impossible to assign alternating signs to five atoms in a ring.

[12] A.J. Lichtenberg, M.A. Lieberman, Regular and Chaotic Dynamics, 2nd edition (Springer-Verlag, New York 1992)

[13] Our increment was $10^{-6}$, the stability matrix was insensitive to changes in the increment.

[14] K.W. Sandusky, J.B. Page, K.E. Schmidt Phys. Rev. B46 6161 (1992)

[15] S. Aubry Physica D in press (1997) 